\title{
Monitoring and visualization techniques for MR- guided laser ablations in an open MR system
}

\section{Citation}

Kettenbach, Joachim, Stuart G. Silverman, Nobuhiko Hata, Kagayaki Kuroda, Pairash Saiviroonporn, Gary P. Zientara, Paul R. Morrison, et al. 1998. "Monitoring and Visualization Techniques for MR-Guided Laser Ablations in an Open MR System." Journal of Magnetic Resonance Imaging 8 (4) (July): 933-943. doi:10.1002/jmri.1880080424.

\section{Published Version}

10.1002/jmri.1880080424

\section{Permanent link}

http://nrs.harvard.edu/urn-3:HUL.InstRepos:33884320

\section{Terms of Use}

This article was downloaded from Harvard University's DASH repository, and is made available under the terms and conditions applicable to Other Posted Material, as set forth at http:// nrs.harvard.edu/urn-3:HUL.InstRepos:dash.current.terms-of-use\#LAA

\section{Share Your Story}

The Harvard community has made this article openly available.

Please share how this access benefits you. Submit a story.

\section{Accessibility}




\title{
Monitoring and Visualization Techniques for MR-Guided Laser Ablations in Open MR-System
}

\author{
Joachim Kettenbach $\mathrm{MD}^{\mathbf{1}}$, Stuart G.Silverman $\mathrm{MD}^{\mathbf{1}}$, Nobuhiko Hata $\mathrm{MS}^{\mathbf{1}}$, Kagayaki Kuroda $\mathrm{PhD}^{4}$, \\ Pairash Saiviroonporn $\mathrm{PhD}^{1}$, Gary P. Zientara $\mathrm{PhD}^{\mathbf{1}}$, Paul R. Morrison $\mathrm{MS}^{\mathbf{1}}$, Stephen G.Hushek $\mathrm{PhD}^{\mathbf{3}}$, \\ Peter McL.Black MD, $\mathrm{PhD}^{2}$, Ron Kikinis $\mathrm{MD}^{1}$, Ferenc A. Jolesz MD ${ }^{1}$
}

Harvard Medical School and Brigham and Women's Hospital:

- ${ }^{1}$ Department of Radiology

- 2 Division of Neurosurgery, Department of Surgery, 75 Francis Street, Boston, MA 02115, USA

${ }^{3}$ General Electric, Medical Systems, Milwaukee, WI

${ }^{4}$ Department of Electrical Engineering, Faculty of Engineering, Osaka City University, Japan

Corresponding author:

Joachim Kettenbach, MD

Dept. of Radiology/MRI

Brigham and Women's Hospital

75 Francis Street, Boston, MA 02115, USA

Tel: (617) 732-5961

Fax: (617) 732-7963

email: kettjo@bwh.harvard.edu

Acknowledgment of support:

This work was supported by: Austrian Science Fund, Project Jo 1315-MED Presented in part at the 5th ISMRM meeting, Vancouver April 1997

\begin{abstract}
Our purpose was to develop temperature sensitive MR sequences and image-processing techniques to asses their potential of monitoring interstitial laser therapy (ILT) in brain tumors $(n=3)$ and liver tumors $(n=7)$. ILT lasted 2 to 26 minutes, while images from T1-weighted Fast-Spin-Echo (FSE)- or Spoiled Gradient-Recalled (SPGR) sequences were acquired within 5 - 13 seconds.
\end{abstract}

Pixel subtraction and visualization of T1-weighted images or optical flow computation was done within less than 110 msec. Alternating, phase-mapping of real- and imaginary components of SPGR sequences was performed within 220 msec.

Pixel subtraction of T1-weighted images identified thermal changes in liver and brain tumors but could not evaluate the temperature values as chemical-shift based imaging, which was however, more affected by susceptibility effects and motion. Optical flow computation displayed the predicted course of thermal changes and revealed that the rate of heat deposition can be anisotropic, which may be related to heterogeneous tumor structure and/or vascularization.

Key words: Magnetic resonance imaging, interstitial laser therapy, optical flow, temperature sensitive imaging, minimally invasive therapy, brain tumor, liver tumor

- $\underline{\text { Introduction }}$

- Materials and Methods

- $\underline{\text { Results }}$

- Discussion

- Conclusion 


\section{- $\quad$ References}

- Tables

- Table 1: Laser parameters and imaging sequences

- Table 2: Sequential processes during pixel subtraction

- Table 3: Laser effects on tissue

- $\quad$ Figures

\section{Introduction}

MR-guided interstitial laser therapy (ILT) is a potentially useful minimally invasive technique. To treat soft tissue tumors without damaging the surrounding structure and in the case of the brain functionally critical tissue, it is important to optimize dose and the position of the optical fiber. The entire tumor volume should be treated with temperatures above $60^{\circ} \mathrm{C}$, the threshold for protein denaturation, but should not exceed $100^{\circ} \mathrm{C}$ since vaporization and charring can cause remote and unpredictable damage. In order to minimize potentially harmful effects and to maximize tissue destruction, low power density and continuous, relatively long duration of deposition of laser energy are preferred. Models, simulating the procedure are not fully applicable because many independent variables which affect heat deposition (vascularity, tissue homogeneity, optical or thermal properties) of normal and tumor tissue cannot be predicted or measured precisely. Multiple invasive probes (thermocouples or temperature-sensitive fiber-optic devices) can be used for direct measurement of local tissue temperature but their spatial resolution is limited. It has been demonstrated that the following MR parameters are temperature dependent: proton density, T1, diffusion, and water proton chemical shift [1-7]. Based on this temperature sensitivity MRI techniques were suggested and tested for the monitoring of hyperthermia and thermal ablations. Recently, temperature-sensitive MR imaging sequences have been developed that acquire images on time scales appropriate for the real-time volumetric monitoring, documenting thermal changes in vitro, in animals or in humans [ $\underline{8}$ $\underline{-21]}$.

The motivating hypothesis of monitoring ILT with temperature-sensitive sequences (T1-weighted FSE or SPGR) and postprocessing techniques described in this study, was to map temperature-related effects dynamically and sufficiently close to real-time, even in an open MR-System. Achieving this goal, the tissue damage throughout the affected tissue volume could be estimated intraoperatively and the ILT then be adjusted.

\section{Materials and Methods}

Approved by the institutional research committee, data were derived from two ongoing clinical trials in our Department, evaluating MRI-guided ILT of malignant liver and brain tumors.

These clinical trials were performed in an $0.5 \mathrm{~T}$ open configuration MR imaging system (SIGNA, SP, GE Medical System, Milwaukee, Wis), in which biopsy, laser fiber placement, laser treatment, and image-based monitoring and control of the various laser therapies were all conducted during the same procedure [22]. All patients in this study provided witnessed informed consent prior to participation.

We assessed MRI monitoring of thermal ablations using preliminary data from 4 patients undergoing liver tumor therapy $(n=7)$, and 3 patients undergoing brain tumor therapy $(n=3)$.

All but one patient with liver tumor were treated under general anesthesia so as to minimize respiratory motion and to obtain long breathhold acquisitions of up to two minutes. All patients with brain tumors were treated under general anesthesia, and the patient s head motions were restricted by a modified Mayfield clamp (Ohio Medical Instruments, Cincinnati, $\mathrm{OH})$.

Laser treatments were performed using a continuous wave $1064 \mathrm{~nm}$ (Nd:YAG) laser (Sharplase 60, Sharplan Laser, Allendale, $\mathrm{NJ})$, and $0.6 \mathrm{~mm}$ bare tip fibers.

In vivo, the calculated penetration depth of an Nd:YAG laser in native human tissue can be expected to be at least $6 \mathrm{~mm}$ [23]. This corresponds to an ellipsoid lesion of about $12 \mathrm{~mm}$ in diameter around the exposed tip of the optical fiber.

Therefore, we used a single fiber for small tumors; if the diameter of the tumor was larger than $12 \mathrm{~mm}$, the tip of the single fiber was either placed at multiple positions and a second or third treatment was applied through the same fiber or multiple fibers were advanced to ablate the entire lesion (Figure 1a) 
Brain lesions were treated with up to 6 irradiations (3-4.5 W), each lasting for 1 minute with a single fiber in place, withdrawn between the irradiations. Liver lesions were treated with up to five irradiations $(2 \mathrm{~W})$, first 8 minutes with a single fiber in place, then $2 \times 8$ minutes with 2 fibers in place.

\section{Imaging During ILT}

Imaging and image-processing involved a conventional SIGNA-SP MR console and the MR workstation (Sparc Station 20, Sun Microsystems, Mountain View, CA). In addition, a research computer workstation (Sparc 20 TZX, Sun Microsystems) was used and we also utilized the local computer network for further computation (Figure 1b).

Each single irradiation was monitored either by a FSE-, or a SPGR sequence. To optimize our MR protocol and the image postprocessing techniques we applied FSE-, or SPGR sequences in some patients only. Occasionally fast SPGR (FSPGR) sequences were applied, but they were not included in this evaluation (Table 1).

Imaging was initiated 30 seconds before ILT to obtain 2-4 baseline images. Then we continued imaging until the end of ILT and took another set of 2-4 images during the cooling down period. In liver treatment the anesthetist suspended respiration during each image acquisition.

\section{a) Pixel Subtraction of T1-weighted Pre-, Intra- and Postoperative Images}

T1-weighted FSE images (TR/TE 400/18 ms, echo-train length 4, slice thickness $5 \mathrm{~mm}$, field of view ((FOV), $220 \times 220$ $\mathrm{mm}$, matrix $256 \mathrm{x} 128,1$ acquisition), were taken every 13 seconds at the tip either perpendicular or in the same plane as the laser fiber.

The sequentially acquired images were then transferred from the image buffer of the MR console via the Ethernet network to the research computer workstation, placed beside the MR console.

For rapid computation, this research workstation was equipped with two fast graphic cards and a fast network communication . For computation and visualization management during ILT, an interactive graphical user interface was created by our group, using $\mathrm{Tk} / \mathrm{Tcl}$, a program written in combination with $\mathrm{C} / \mathrm{C}++$ programming languages [24]. To highlight the decrease in SI on T1-weighted images during ILT, a pixel subtraction was performed, which consisted of sequential processes, described in Table 2. The final color-encoded result images were displayed during laser therapy on both the monitor of the workstation and on a liquid-crystal display (LCD, Model LQ6NC01,Sharp Electronics, Rahwah,NJ,USA) inside the magnet within seconds delay (Figure 2).

\section{b) Pixel Subtraction of T1-weighted Pre-, Intra- and Postoperative Images with a Real-Time Image Processor}

A prototype software package was developed and provided by GE Medical Systems for the interventional MR workstation connected to the conventional SIGNA MR console. This MR workstation performed algorithmic combinations of T1-weighted FSE images (similar to the method described above) immediately as they were transferred from the MR console. The images resulting from such calculations were then displayed in color over a grayscaled anatomical image. Following the processing we masked and averaged the signal intensity (SI) changes in time series within regions of interests (ROIs) at different distances from the tip of the optical fiber (Figure 3a,b)

With each new image acquisition, the averaged SI changes (subtracted from the baseline) were color-encoded and their amount plotted as data points on graph bars.

\section{c) Calculation of water proton chemical shift change and phase-mapping during ILT}

The MR scanner was programmed to reconstruct real, and imaginary images from the SPGR pulse sequence (TR/TE $55 / 14 \mathrm{~ms}, 20^{\circ}$ flip angle, slice thickness $4 \mathrm{~mm}, 1.5 \mathrm{~mm}$ gap, FOV $320 \times 240 \mathrm{~mm}$, matrix 256 x 128, 1 acquisition). To cover the whole tumor volume, images in one to four different scan planes parallel to the laser fiber were obtained. Those images were transferred to the research workstation for temperature image processing. In [25], Kuroda et al proposed the 


$$
\Delta \delta=\frac{\Delta \phi}{\omega_{\mathrm{RF}} \cdot T E}
$$

$$
\Delta \phi=\tan ^{-1}\left(\frac{\operatorname{Re}\left[S\left(T_{B}\right)\right] \cdot \operatorname{Im}[S(T)]-\operatorname{Re}[S(T)] \cdot \operatorname{Im}\left[S\left(T_{B}\right)\right]}{\operatorname{Re}[S(T)] \cdot \operatorname{Re}\left[S\left(T_{B}\right)\right]+\operatorname{Im}[S(T)] \cdot \operatorname{Im}\left[S\left(T_{B}\right)\right]}\right)
$$

whereas is the angular frequency of the $0.5 \mathrm{~T}$ MR-scanner $\left(2 \pi \times 21.3 \times 10^{6} \mathrm{rad} / \mathrm{s}\right), \Delta \phi$, is the phase distribution difference between the objective-temperature $(\mathrm{T})$ image and the baseline-temperature $\left(\mathrm{T}_{\mathrm{B}}\right)$ image, $S$ is the

complex MR signal, and Re [] and Im []w 0 RF $\left(2 \pi \times 21.3 \times 10^{6} \mathrm{rad} / \mathrm{s}\right), \Delta \phi$ is the angular frequency of the $0.5 \mathrm{~T}$ MR-scanner image and the baseline-temperature $\left(\mathrm{T}_{\mathrm{B}}\right)$ image, is the complex MR signal, and $\operatorname{Re}[]$ and $\operatorname{Im}[]$ denote the real and imaginary parts, respectively. To avoid phase wrapping, a direct (phase)subtraction, as used for the pixel subtraction in step 3 of Table 2 was replaced by the complex subtraction of real and imaginary components of the MR signal (described

in equation 1 and 2). To maximize the signal to noise ratio for a given TR and T1, the optimal flip angle

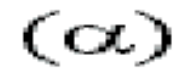
was determined by the Ernst relation as follows:

$$
\begin{gathered}
\alpha=\cos ^{-1}\left(\exp \left(\frac{\mathrm{TR}}{\mathrm{T} 1}\right)\right. \\
\Delta \operatorname{temp}\left({ }^{\circ} \mathrm{C}\right)=\frac{\Delta \phi}{2 \pi \cdot \omega \mathrm{RF} \cdot T E \cdot a}
\end{gathered}
$$

The temperature elevations were calculated as described in (4) with Matlab 4.2a (MathWorks, Natick, MA), whereas a is the temperature coefficient of $-0.01 \mathrm{ppm} /{ }^{\circ} \mathrm{C}$.

Given that the temperature coefficient of tissue in vivo would be around $-0.01 \mathrm{ppm} /{ }^{\circ} \mathrm{C}$ the relative temperature elevation during ILT could be measured. By adding the relative temperature elevation to the body temperature, which can be assumed to be $37^{\circ} \mathrm{C}$ absolute temperature values were obtained. For visualization of the phase differences an interactive display was developed (Figure 4).

\section{d) Optical Flow Computation and Display}

Optical Flow (OF) is the motion field perceived by the human visual system when exposed to a set of time-varying image frames (i.e. an image sequence). OF can also described as an instantaneous velocity vector field of moving isointense image regions, computed from a time series of images.

Sequentially acquired T1-weighted FSE images were transferred via the network to the Surgical Planning Laboratory (SPL), where the optical flow computation was performed. Uras et al. proposed an efficient method to calculate the OF field and by using a parallel computer (Connection Machine 2, Thinking Machine Corp., Cambridge MA) this allowed fast calculation of the optical flow in two dimensions [26-29]. 
As the ILT proceeded, the continuously dilating marginal zone of heated tissue (seen as isointense region) was tracked at each time step. This zone was determined by a sequential pixel subtraction from the baseline image. Immediately following an actual image, a new prediction of heat distribution was computed. Assuming constant velocities of the heat distribution between image-acquisitions, therefore the predicted amount (OF magnitude) and the direction (OF velocity vectors) of heat deposition was obtained and superimposed in color on the gray scale image (Figure 5). The resulting dynamic images were then transferred back to the research workstation in the interventional MR suite for direct visualization.

Following ILT, patients underwent clinical follow-up imaging at a $1.5 \mathrm{~T}$ scanner. T1-weighted SE, T2-weighted FSE, T1weighted SE post Gd-DTPA, fast multiplanar SPGR with breathhold in liver cases were obtained the next day, and in some cases in 3, and 6 months (iure 6 ). The size and the structure of the treated lesions was compared with that before treatment by direct visualization.

As an clinical example we measured the SI-distribution within a brain lesion before, during and after treatment.

\section{Results}

Within the open configuration MR system and by means of interactive image guidance [22], the laser fibers were placed easily through a MR-compatible biopsy needle into the tumor. The total energy applied in all brain tumors was in the range of $720-1440 \mathrm{~J}$, and for all liver tumors in the range of $960-4800 \mathrm{~J}$.

\section{Imaging and Image Processing}

\section{Pixel subtraction of T1-weighted images}

With the currently used sequence (TR $400 \mathrm{~ms}, 128$ phase encoding steps, echo train length 4) for both techniques (described in the method section as a and b) the image acquisition time was about $13 \mathrm{sec}$. This included the image data transfer and the computation time for steps 2-7 in Table 2, which took in particular about $27 \mathrm{~ms}$ ). Pixel subtraction of T1weighted images was easily performed with current available hardware and our self-developed software, providing a (screen-based) easy-to-use interactive user interface.

The prototype software (provided by GE Medical System) was more flexible for user-defined changes in calculation and visualization. Especially, we could plot the amount of SI-values, whereas the values of each single image acquisition were represented by a white marker on a graph bar. However, many variables had to be defined and, therefore, more interaction was needed to handle the interface. With both image processing techniques, we localized temperature-related changes within brain and liver tumor. A gradually increasing elliptical zone of decreasing signal intensities, expanding from the tip of the laser fiber was highlighted as color-encoded pixels for each series of ILT. The color encoded pixels were seen first 13 seconds after starting ILT and increased to a maximum diameter of 6-8 $\mathrm{mm}$ at the end of ILT (Figure $2, \underline{3}$ ).

The quality of temperature-sensitive imaging with T1-weighted FSE sequences (seen as a significant reduction in signal intensity values within treated tissue) was higher in the brain than in the liver. This can be explained by the larger field inhomogeneity in liver imaging and by the presence of unavoidable motion during liver treatment, even if the respiration was suspended.

Intermittent pausing of respiration under general anesthesia during laser delivery significantly improved image quality but not completely eliminated fully organ shifts and motions.

When the result images were still too noisy for visual evaluation, we masked and averaged regions of interest in time series with the prototype software (provided by GE Medical System) and compared the graphs of temperature-related signal intensity changes at different distances from the tip of the optical fiber ( $\underline{\text { Figure } 3 a, b}$ ).

\section{Water proton chemical shift imaging and phase mapping}

The real and imaginary images were retrieved from the MR console within $5-7$ seconds for each slice, and the computation time for one resultant image took an additional $220 \mathrm{~ms}$. Temperature related changes correlated with the time course of ILT and were first seen 10 seconds after starting ILT. The phase shift differences (respectively the temperature elevation) increased to a maximum value at the end of ILT. The calculated maximum temperature elevation within the 
brain tumor during ILT with 3 Watt for 1 minute (i.e. $180 \mathrm{~J}$ ) was about 15 - 20 degree Celsius (Figure4) above body temperature. The calculated maximum temperature elevation within the liver tumor during a single fiber treatment (2 Watt for 8 minutes i.e. $960 \mathrm{~J}$ ) was about 45 degree Celsius above body temperature. However, in liver treatment motion and artifacts partially covered the region were the laser fiber has been placed.

\section{Optical Flow Computation and Display}

OF computations were performed within an execution time of $110 \mathrm{msec}$ for $256 \mathrm{x} 256$ pixel images, with the capability of updating the display of optical flow results every $900 \mathrm{msec}$ as soon as the T1-weighted FSE images were retrieved from the MR scanner. Therefore OF information was much earlier available than with any other postprocessing method. The OF computation revealed how laser energy changed with time, directing radially from the laser fiber and that the heat deposition within the tumor was not at the same rate at each location which may be related to inhomogenous tumor structure and/or vascularization. (Figure 5). This unique information was not seen from the signal intensity display alone.

On this study, all OF computations were performed after the procedure following the complete image series acquisition because the Connection Machine in the SPL was not fully integrated for computation during the ILT procedure.

The days following ILT, in all imaging studies we found a low signal intensity zone on dynamic T1-weighted sequences, hyperintense on T2-weighted images, that (as previous experiments and studies have shown in 13,17,21,30,36) corresponded to tissue necrosis within the tumor (Figure 6).

The overall size of the necrosis on images following ILT was slightly larger than on the color-encoded area of pixel subtraction during ILT (Figure 7). However, postprocessing of fast MRI during ILT visualized larger thermally affected areas than MRI within few minutes after the procedure.

Compared with the thermally affected area on chemical shift-based imaging, the size of the necrosis following ILT was almost the same. Based on T1-weighted contrast, OF computation slightly underestimated the size of necrosis. However, with this method thermal changes during ILT could be predicted to prohibit damage of critical structures, which otherwise would not be recognized in time. A liver specimen was obtained from one patient who recently underwent partial hepatectomy. Histology confirmed a fully necrotic tissue around the formerly placed laser fibers. A 3-4 mm thick fibrotic rim could be seen between the necrosis and surrounding liver tissue. and surprisingly, a small area of central charring has been found within the treated area. (Figure 8)

\section{Discussion}

Our objective was to develop and compare temperature sensitive MR sequences and image-processing techniques for clinically monitoring ILT, even in an open MR configuration.

It has been demonstrated by us and others that temperature-related effects could be mapped dynamically sufficiently close to real time, throughout the affected tissue volume $[4-7, \underline{9-21}, \underline{30}]$. The results show that temperature sensitive MRI can monitor transient thermal effects (including heat loss in blood vessels) and irreversible tissue changes during ILT with the postprocessing techniques described. In addition it has been shown, that fast MRI visualized significantly larger thermal affected lesions and irregular heat distribution than MRI within few minutes after ILT, which may change treatment strategies, and potentially provide intraoperative control methods [이] . Further, an accurate measure of laser treatment volumes during ILT is essential for MRI-guided therapy.

Nevertheless, physiologic and metabolic responses of treated tissues to increased temperature could modify the temperature-sensitivity of MRI parameters. There may be other factors such as tissue type, pigmentation, vascularity that could produce changes in image signal intensity, whereas nature, magnitude, and interplay of these factors is not well known.

One example of this is the change from hemoglobin to methhemoglobin at approximately $60^{\circ} \mathrm{C}$ and the associated shortening of $T 1$ relaxation time. Furthermore, there are also temperature induced changes in tissue perfusion and several complex metabolic, structural and physiological changes with increasing temperature (Table 3 ).

Comparison of image sequences and techniques 
Diffusion sequences [4-6, 13] have a relatively high sensitivity ad therefore may be used for temperature mapping. But the enhanced temperature sensitivity in diffusion imaging comes at the expense of a low signal-to-noise ratio in the images an requires fast or ultrafast imaging methods to avoid the effect of macroscopic motion. We therefore did not use diffusion sequences in this study.

Studies in our group [9-11, 13-21] and clinical trials [44-46] have shown that relatively fast T1-weighted protocols and SPGR sequences are useful for monitoring thermal changes in tissue. The combination of higher signal levels and faster scans compensates for the lesser temperature contrast, which can be enhanced with the image processing techniques described in this publication.

\section{Pixel subtraction of T1-weighted images}

Pixel subtraction of T1-weighted images can be performed easily with currently available computer hard- and software. To visualize the margins of laser-induced lesions during ILT, this method revealed temperature-related SI-changes, especially in isointense lesions (liver) which are not clearly visible otherwise.

Matsumoto et al $[\underline{9}, \underline{13}]$ has shown that within the range of $37-50^{\circ} \mathrm{C}$, the relaxation time $T 1$ varies approximately linearly with temperature at a rate of $1 \% /{ }^{\circ} \mathrm{C}$. Thus, MRI can distinguish the normal margins surrounding thermal lesions. However, when heated above $50^{\circ} \mathrm{C}$ the T1-dependence on temperature varies in different tissue types and deviates from the linear behavior due to physiological, structural, and metabolic changes in the tissue during thermal therapy exposure.

Those factors and some residual motion may have been the reason why we underestimated charring in one liver case. Pixel subtraction is not as sensitive to temperature related changes as chemical-shift based-imaging, but is sensitive to any movement of tissue within the field-of-view.

\section{Water proton chemical-shift imaging and phase mapping}

Chemical-shift based imaging is reported to be more temperature-sensitive when combined with phase mapping than T1 weighted imaging with FSE sequences $[\underline{31}, \underline{32}]$. This technique of noninvasive MRI thermometry has been recommended for several reasons:

1. The water proton chemical shift is a frequency-based parameter and can be measured independently from other amplitude-based parameters such as thermal equilibrium magnetization, relaxation time and diffusion coefficient

2. The water proton signal is much stronger than that of any other components and is measurable in most tissues

3. The temperature sensitivity of pure water has been measured to be $-0.01 \mathrm{ppm} /{ }^{\circ} \mathrm{C}$, which is of the same order as animal tissues in vitro $[\underline{1}, \underline{2}]$.

The temperature sensitivities of human tissues in vivo, however are not well known yet. Although the temperature elevation estimated from the phase map seemed to be close to the actual value ( 15 degree Celsius within a brain tumor, 45 degree Celsius within liver tumor) no evidence was made in the present series of irradiations. However, based on previous experiments $[\underline{20}, \underline{21}, \underline{32}]$ the possible uncertainty of estimated temperature will be around $20 \%$. The lower elevation of temperature in brain treatment may not only be due to the low laser power applied, but also due to the fact that the $\mathrm{Nd}$ :YAG wavelength is best absorbed and scattered in red-pigmented tissue, such as liver.

A major drawback is that water proton chemical-shift imaging is more sensitive to motion. In liver treatment this was expressed by a high amount of noise on the temperature-graphs and (false positive) color-encoded signal intensities. They were present within the field-of-view even without treatment, enhanced by tissue motion, field inhomogeneities, or other susceptibility related artifacts.

Further optimization of SPGR will be necessary in future treatments to improve SNR which can be reduced by flow related artifacts in the sinusoids of liver, and the release of paramagnetic substances from liver cells during ILT.

The echo time $T E$ may be optimized to increase the phase contrast-to-noise ratio. While the phase shift increases with $T E$, the magnitude signal decreases with increasing $T E$ because $T_{2} *$ effects reduce the magnitude signal. Described by Cline [18] and by Chung et al [19] the maximum temperature sensitivity can be achieved when $T E$ is around the $T_{2}^{*}$ value of treated tissue, which we recently measured to be around $34 \mathrm{~ms}$ for liver tissue. Next it would be optimal to use the longest $T R$ possible; however, to reduce the imaging acquisition time to 5 - 7 seconds and to minimize motion artifacts during acquisition a $T R$ of $50 \mathrm{~ms}$ was used. 
To minimize further motion artifacts during imaging of ILT in liver, breathhold acquisition is mandatory. However, even under general anesthesia and breathhold acquisitions lasting from 15 seconds up to 2 minutes there was always some slight deviation of the liver's position during ILT compared with baseline images. Therefore, some type of respiratory gating, navigator pulse sequences [33], or interactive in-plane imaging using motion tracking coils [34] could be useful for imaging of moving tissues .

\section{Optical-Flow computation}

Analysis of OF provides new and important information about heat deposition very close to real-time and a spatiotemporal description of the thermal changes even when the treated anatomy contained heterogeneous tissue (e.g., cerebral and tumorous tissue) and vessels. Moreover, analysis of two-dimensional (2D) OF predicts deposition and velocity of thermal ablation to adjacent tissues, which can be very inhomogeneous. This important information is not provided by other methods.

A distinct advantage of this approach is its applicability to three-dimensional (3D) monitoring which was not applied on this study. 3D OF and treatment zone forecasts can be radially computed using 3D dynamic MRI data, and can be used for automated feedback control of heat deposition, that ensures the safety and effectiveness of thermal ablations. However, when the intensity of the difference image is lower than the noise and when tissue deforms due to thermal effect, OF is not practical. Although OF used a parallel multiprocessor workstation for fast image processing, on a regular workstation a 2D OF computation would be available, within 10- 15 seconds.

The size of the necrosis on images taken the day following ILT was slightly larger than on the color-encoded thermal changes seen during monitoring ILT with pixel subtraction. This can be explained by a cumulative effect of ILT [ $\underline{35}, \underline{36}]$, because ILT was performed for up to 5 time series, each serie within the range of 1-8 minutes. Furthermore, in single fiber treatment, the fiber was withdrawn or multiple fibers were placed within the tumor to enlarge the treated area.

In addition, the fact that thermal ablation in vivo is affected by thermal conductivity and convection by blood flow must be considered, which is neither significantly dependent on the species nor on the specific organ. Although the given in vivo values depend on the rate of perfusion and can be up to three times higher [37]. In the future this will require software similar to the laser simulator [38], that takes the accumulative factor of heating into account, which has not been integrated into our system.

Since we modified our treatment-protocol and the post-processing techniques, a true comparison of ILT with this preliminary results is not obtainable. Although, our initial clinical results demonstrate the feasibility of MR-guided ILT and the advantages and disadvantages in temperature-sensitive monitoring. In particular, due to the lack of movement, thermal monitoring in brain is much easier to perform than in liver.

\section{Role of "Contrast enhancement"}

It should be taken into account, that Gadolinium-DTPA, applied to enhance tumor visualization, provides a positive susceptibility component. Therefore, the calculated temperature values in chemical shift-based imaging during ILT will be smaller.

Paramagnetic thermometric complexes (Tm-DOTP5-) significantly improve the temperature sensitivity in chemical-shift based imaging. But due to their toxicity for tissue they will not contribute to clinical monitoring thermal ablations in the near future [우].

To reduce the risk of charring or vaporization and to increase the treated lesion size heat-resistant laser-applicators, recently described by Vogl et al [40], or cylindrical diffusing laser fibers [41] should be used. They can lead to coagulative necrosis with a nearly spherical shape of up to $2 \mathrm{~cm}$ radius without central charring. Therefore, tumors with a diameter of 3-4 cm can be treated over their entire diameter in one session.

Spectroscopic imaging, combined with fast (echoplanar) imaging techniques might be advantageous to overcome susceptibility artifacts during ILT. Using the fat resonance as an internal reference this method does not require subtraction techniques. Based on preliminary experiments the 2D distribution of the proton spectrum can be taken in 10 sec with Echo-Planar Spectroscopic-Imaging (EPSI) in $24 \mathrm{sec}$ [42]. 


\section{Conclusions}

MR-guided ILT can provide an effective delivery of thermal energy to tissue with minimal damage to adjacent structures and thus became a potential method of treating different types of localized tumors in liver [43, 44], head and neck [45], brain [ $\underline{8}, \underline{46}]$ and breast [47]. Particularly for deep malignant cerebral lesions, usually inaccessible with surgery, or in lesions not otherwise visualized MRI-guided ILT offers a successful treatment option. Although access to the patient and fiber placement was performed more easily in the open magnet than it would have been in a closed MRI system, monitoring of ILT with the described techniques could be implemented in almost every MRI system. All of the applied image processing methods enhanced visualization of temperature-related effects and displayed them near real-time. Although with all the methods described, real-time processing thus has not yet been achieved, they are adequate and valuable for clinical ILT with a duration of up to 26 minutes.

Optimum monitoring sequences and post-processing techniques

Appropriate interpretation of intraoperative images is critical to the development of a control system for thermal surgeries (Table 4).

1. Pixel subtraction of T1-weighted MRI has limitations as an exact "tissue thermometer" and therefore it is ineffective for characterizing the extent of cell damage. The MRI signal intensity may reveal the presence of tissue phase transition and monitor the leading edge of a temperature gradient moving toward nearby structures. This effect is especially useful in the OF computation

2. Chemical shift-based imaging with phase mapping promises a higher temperature sensitivity and semiquantitative temperature measurements within a laser-induced lesion. However, due to many artifacts monitoring of liver treatment needs more improvements.

3. 2D-Optical Flow computation and visualization is easily attainable, successfully forming the basis of a computerized automatic control software during ILT. A distinct advantage of this approach is its applicability to 3D monitoring. Again, OF computation cannot measure temperature and is not practical when tissue movement or deformation occurs.

There will be a need for a more reliable thermometry in vivo, prediction of tissue necrosis in all three dimensions as well as considerable improvements in monitoring, particularly in moving tissues. At that time, MR-guided ILT may have a major impact on minimally invasive treatment.

\section{Acknowledgment}

This work was supported by the Austrian Science Fund (Project Jo-1315 MED), Program project grant support in part (NIH P01CA6716503), and a laser grant (NSF BES-9631710).

We are very grateful for creating the interface for the phase-mapping method of Mr. Yoshikazu Nakajima of the University of Osaka and for the contributions and certain technical support for this study, provided by Nathan J.

McDannold, BS (Image Guided Therapy,Focused Ultrasound Group, Harvard Medical School), Dan Kacher, MR engineer (Surgical Planning Laboratory, Brigham and Women's Hospital), Ray Kelley, BS (GE Medical Systems) and Hugh L. Flanagan Jr. (Dept. of Anesthesiolgy, Brigham and Women's Hospital).

\section{References}

[1] Parker DL, Smith V, Sheldon P, Crooks LE, Fussel L Temperature distribution measurements in two dimensional NMR imaging. Med Phys.1983;10:321-325

[2] Bottomley PA, Foster TH, Argersinger RE, Pfeifer LM. A review of normal tissue hydrogen NMR relaxation times and relaxation mechanisms from 1 - 100 Mhz: dependence on tissue type, NMR frequency, temperature, species, excision and age. Med.Phys 1984;11:425-448 
[3] Dickinson RJ, Hall AS, Hind AJ, Young IR Measurement of changes in tissue temperature using MR imaging J Comput. Assist. Tomogr. 1986;10:468-472

[4] Jolesz FA, Bleier AR, Jakab P, Ruenzel PW, Huttl K, Jako GJ MR imaging of laser-tissue interactions Radiology 1988;168:249-253

[5] LeBihan D, Delannoy J, Levin RL Temperature mapping with MR imaging of molecular diffusion: application to hyperthermia Radiology 1989;171:853-857

[6] Bleier AR; Jolesz FA; Cohen MS; Weisskoff RM; Dalcanton JJ; Higuchi N; Feinberg DA; Rosen BR; McKinstry RC; Hushek SG Real-time magnetic resonance imaging of laser heat deposition in tissue

[7] Ishihara $\mathrm{Y}$, Calderon $\mathrm{A}$, Watabene $\mathrm{H}$ et al. A precise and fast temperature mapping using water proton chemical shift in Proc. SMRM, 11th Annual Meeting,Berlin, 1992 p.4803

[8] Ascher PW,Justich E, Schrottner O Interstitial thermotherapy of central brain tumors with Nd:YAG laser under realtime monitoring by MRI J Clin Laser Med Surg 1991;9:79-83

[9] Matsumoto R, Oshio K, Jolesz FA Monitoring of laser and freezing-induced ablation in the liver with T1-weighted MR imaging. J Magn Reson Imaging 1992;2:555-562

[10] Higuchi N, Bleier AR, Jolesz FA, Colucci VM, Morris JH Magnetic resonance imaging of the acute effects of interstitial Nd:YAG laser irradiation in tissue. Invest Radiol 1992;27:814-821

[11] Hushek SG, Morrison PR, Kernahan GE, Fried MP, Jolesz FA Thermal contours from magnetic resonance images of laser irradiated gels Proc Biomed Optics Eur 1993;2082:52-59

[12] Tracz RA, Wyman DR, Little PB et al. Comparison of magnetic resonances images and histopathological findings of lesions induced by interstitial laser photocoagulation in the brain. Laser Surg Med 1993;13:45-54

[13] Matsumoto R, Mulkern RV, Hushek SG, Jolesz FA Tissue temperature monitoring for thermal interventional therapy: comparison of T1-weighted MR sequences J Magn Reson Imaging 1994; 4:65-70

[14] Cline HE, Hynynen K, Hardy CJ, Watkins RD, Schenck JF, Jolesz FA MR temperature mapping of focused ultrasound surgery Magn Resons. Med 1994;31:628-636

[15] Magnetic Resonance-guided thermal surgery MRM 1993;30:98-106

[16] Ishihara Y, Calderon A, Watabene $\mathrm{H}$ et al. A precise and fast temperature mapping using water proton chemical shift Magn. Reson. Med. 1995;34:814-823

[17] Fried MP Morrison PR, Hushek SG, Kernahan GA, Jolesz FA Dynamic T1-weighted magnetic resonance imaging of interstitial laser photocoagulation in the liver: observations on in vivo temperature sensitivity. Lasers Surg Med (United States), 1996, 18(4) p410-9

[18] Cline HE; Hynynen K; Schneider E; Hardy CJ; Maier SE; Watkins RD; Jolesz FA Simultaneous magnetic resonance phase and magnitude temperature maps in muscle Magn Reson Med 1996 Mar;35(3):309-15

[19] Chung AH, Hynynen K, Colucci V, Oshio K, Cline HE, Jolesz FA Optimization of Spoiled Gradient -Echo phase imaging for in vivo localization of a focused ultrasound beam MRM 1996;36:745-752

[20] Kuroda K, Chung AH, Hynynen K, Jolesz FA Calibration of water proton chemical shift with temperature for noninvasive temperature imaging during focused ultrasound surgery Proceedings of 5th ISMRM, Vancouver 1997:p.266

[21] Hynynen K,Vykhodtseva N.I, Chung A.H., Sorrentino V, Colucci V., Jolesz FA Thermal effects of focused ultrasound on the brain: determination with MR imaging Radiology 1997;204:247-253

[22] Silverman SG; Collick BD; Figueira MR; Khorasani R; Adams DF; Newman RW; Topulos GP; Jolesz FA Interactive MR-guided biopsy in an open-configuration MR imaging system Radiology 1995;197:175-181, Comment in: Radiology

1995 Oct; 197(1):16-8 
[23] Roggan A, Dörschel K, Minet O, Wolff D, Müller G The optical properties of biological tissue in the near infrared wavelength range-review and measurementsLaser-induced interstitial thermotherapy editors: Müller G.J., Roggan A.published by SPIE-The International Society for Optical EngineeringBellingham Washington, 1995,p. 10-44

[24] John K.Ousterhout, Tcl and the Tk Toolkit, Addison-Wesley Publishing 1994, ISBN 0-201-63337-X[25] Kuroda K, Suzuki Y, Ishihara Y, Okamoto K, Suzuki Y Temperature mapping using water proton chemical shift obtained with 3DMRSI: feasibility in vivo Magn Reson Med 1996;35:20-29

[26] Uras S, Girosi F, Verri A, Torre V A computational approach to motion perception Biol.Cybern. 1988; 60: 79-87[27] Rogers B Perspectives on movement Nature 1988;333:16-17

[28] Zientara GP, Saiviroonporn P, Kikinis R, Jolesz FA Control of MRI-monitored thermal therapies using optical flow Proceeedings of 3th SMR, Nice 1995, p. 1179

[29] Amartur SC, Vesselle HJ, A new approch to study cardiac motion: the optical flow of cine MR images Mag Res Med $1993 ; 29: 59-67$

[30] Pushek T, Farahani K, Saxton RE, Saxton RE; Soudant J; Lufkin R; Paiva M; Jongewaard N; Castro DJ Dynamic MRI-guided interstitial laser therapy: A new technique for minimal invasive surgery. Laryngoscope 1995; 105:1245-1252

[31] De Poorter J; De Wagter C; De Deene Y; Thomsen C; Stahlberg F; Achten E Noninvasive thermometry with the proton resonance frequency (PRF) method: in vivo results in human muscle Magn Reson Med 1995; 33:74-81

[32] Kuroda K, Somatani N, Suzuki Y, Ishihara Y, Okamoto K Temperature mapping using water proton chemical shift Proc. 2nd SMR, San Francisco, 1994, p.1569

[33] Harth T, Schwabe B, Kahn T Motion corrected proton-resonance-frequency method for MR-thermometry In Proc. 5th ISMRM, Vancouver 1997, p.1956

[34] Leung DA; Debatin JF; Wildermuth S; Heske N; Dumoulin CL; Darrow RD; Hauser M; Davis CP; von Schulthess GK Real-time biplanar needle tracking for interventional MR imaging procedures. Radiology 1995,197(2) 485-488

[35] McKenzie AL. Physics of thermal processes in laser-tissue interaction Phys Med Biol 1990;35(9):1175-1209

[36] Farahani K, Mischel PS, Black KL, DeSalles AAF, Anzai Y, Lufkin RB Hyperacute thermal lesions: MR imaging evaluation of development in the brain. Radiology 1995; 196:517-520

[37] Giering K, Minet O, Lamprecht I, Müller G Review of thermal properties of biological tissues Laser-induced interstitial thermotherapy editors: Müller G.J., Roggan A. published by SPIE-The International Society for Optical Engineering Bellingham Washington, 1995, p. 45-65

[38] Roggan A, Müller G Dosimetry and computer-based irradiation planning for laser-induced interstitial thermotherapy (LITT) Laser-induced interstitial thermotherapy editors: Müller G.J., Roggan A. published by SPIE-The International Society for Optical Engineering Bellingham Washington, 1995, p. 114-156

[39] Zuo CS, Bowers JL, Metz KR, Sherry Ad MR temperature measurement in vivo with TmDOTP5- In Proc. 5th ISMRM, Vancouver 1997, p.1961

[40] Vogl TJ, Mack MG, Roggan A, Straub R et al. A new design of a percutaneous application set for MR-controlled laser-induced thermotherapy RSNA 1996;p.389

[41] Heisterkamp J, VanHillegersberg R, Sinofsky E, Ijzermans JNM Heat-resistant cylindrical diffuser for interstitial laser coagulation: comparison with the bare-tip fiber in a porcine liver model Lasers in Surgery and Medicine 1997;20:304-309

[42] Kuroda K, Oshio K, Panych LP et al Preliminary experimental results with multiple-shot EPSI submitted to ISMRI 98

[43] Gewiese B; Beuthan J; Fobbe F; Stiller D; Muller G; Bose-Landgraf J; Wolf KJ; Deimling M Magnetic resonance imaging-controlled laser-induced interstitial thermotherapy Invest Radiol 1994;29(3):345-51 
[44] Vogl TJ; Muller PK; Hammerstingl R et al Malignant liver tumors treated with MR imaging-guided laser-induced thermotherapy: technique and prospective results. Radiology 1995; 196(1): 257-265

[45] Castro DJ, Lufkin RB, Saxton RE et al. Metastatic head and neck malignancy treated using MRI guided interstitial laser phototherapy: an initial case report. Laryngoscope 1992;102:26-32

[46] Kahn T; Bettag M; Ulrich F et al MRI-guided laser-induced interstitial thermotherapy of cerebral neoplasms. J Comput Assist Tomogr 1995;18(4):519-532

[47] Mumtaz H, Hall-Craggs M, Wotherspoon A et al. Laser therapy for breast cancer: MR imaging and histopathologic correlation Radiology 1996; 200: 651-658

\section{Tables:}

Table 1: Laser parameters and imaging sequences

\begin{tabular}{|c|c|c|c|c|c|c|}
\hline Patient & Localization & \begin{tabular}{|l} 
Laser \\
Output
\end{tabular} & Duration & $\begin{array}{l}\text { Number of } \\
\text { fibers in place }\end{array}$ & $\begin{array}{l}\text { Total Energy } \\
\text { applied (J) }\end{array}$ & $\begin{array}{l}\text { Imaging } \\
\text { Sequence }\end{array}$ \\
\hline A.J. & brain & $\begin{array}{l}4 \mathrm{~W} \\
4 \mathrm{~W} \\
4 \mathrm{~W} \\
4 \mathrm{~W} \\
4 \mathrm{~W} \\
4 \mathrm{~W}\end{array}$ & $\begin{array}{l}1 \mathrm{~min} \\
1 \mathrm{~min} \\
1 \mathrm{~min} \\
1 \mathrm{~min} \\
1 \mathrm{~min} \\
1 \mathrm{~min}\end{array}$ & $1 *$ & 1440 & $\begin{array}{c}\text { FSE } \\
\text { FSPGR } \\
\text { FSE } \\
\text { SPGR } \\
\text { FSE } \\
\text { FSE }\end{array}$ \\
\hline P.L. & liver & $\begin{array}{l}2 \mathrm{~W} \\
2 \mathrm{~W} \\
2 \mathrm{~W}\end{array}$ & $\begin{array}{l}8 \min \\
8 \min \\
8 \min \end{array}$ & $\begin{array}{c}1 \\
2 \\
2 *\end{array}$ & 4800 & $\begin{array}{c}\text { FSE } \\
\text { SPGR } \\
\text { SPGR }\end{array}$ \\
\hline
\end{tabular}

* laser fiber was withdrawn and/or advanced after the second irradiation

Table 2: Sequential processes during pixel subtraction

\begin{tabular}{||c||l||}
\hline \multicolumn{1}{|c|}{ Step } & \multicolumn{1}{|c|}{ Procedure } \\
\hline \hline 1 & Sequential imaging and data transfer to the computer workstation \\
\hline \hline 2 & Definition of a ROI within the FOV of the MR image \\
\hline 3 & Pixel subtraction of images during ILT from the baseline image \\
\hline \hline 4 & $3 \times$ 3 Low-pass Gaussian filtering for noise reduction \\
\hline \hline 5 & Interactive tresholding to remove noise and selectively enhance the heated region \\
\hline \hline 6 & $\begin{array}{l}\text { Color coding of the subtracted images, enhancing the thermal effect by assigning color, based on the signal } \\
\text { intensity (SI) level of the subtraction image (red-high SI, green -middle SI, blue-low SI) }\end{array}$ \\
\hline \hline 7 & Blending of the subtraction image and the (gray-scaled) baseline image if required. \\
\hline \hline
\end{tabular}


Table 3: Laser effects on tissue

\begin{tabular}{|l||l|l||}
\hline Terminology & Temperature & Histopathology \\
\hline \hline Hyperthermia & $42-45^{\circ} \mathrm{C}$ & $\begin{array}{l}\text { Reversible damage to cellular enzymes, may become irreversible after } \\
\text { long exposure time }(>25 \mathrm{~min})\end{array}$ \\
\hline $\begin{array}{l}\text { Coagulation and } \\
\text { vaporization of tissue } \\
\text { water }\end{array}$ & $60-100^{\circ} \mathrm{C}$ & $\begin{array}{l}\text { Protein denaturation, hyalinization of collagen and cell shrinkage } \\
\text { (macroscopically tissue blanching) }\end{array}$ \\
\hline \hline Carbonization and ablation & above $100^{\circ} \mathrm{C}$ & Charring with increased light absorption and smoke generation \\
\hline
\end{tabular}

Table 4: Advantages and disadvantages of imaging and postprocessing techniques

\begin{tabular}{|c|c|c|}
\hline $\begin{array}{l}\text { Imaging Methods and } \\
\text { Postprocessing } \\
\text { Techniques }\end{array}$ & Advantages & Disadvantages \\
\hline \multirow[t]{4}{*}{$\begin{array}{l}\text { Pixel subtraction of T1- } \\
\text { weighted images }\end{array}$} & $\begin{array}{l}\text { Linear relation of } T 1 \text { with temperature } \\
\text { within } 37^{\circ}-50^{\circ} \mathrm{C}\end{array}$ & $\begin{array}{l}\text { Deviation of } T 1 \text { from linear behavior above } \\
50^{\circ} \mathrm{C}\end{array}$ \\
\hline & $\begin{array}{l}\text { Improves visualization of thermal changes } \\
\text { even in tissues with low SI }\end{array}$ & $\begin{array}{l}\text { Temperature dependency of } T 1 \text { is strongly } \\
\text { tissue specific }\end{array}$ \\
\hline & Not sensitive to susceptibility artifacts & Sensitive to motion \\
\hline & $\begin{array}{l}\text { Fast and easy postprocessing with current } \\
\text { available hard- and software }\end{array}$ & $\begin{array}{l}\text { Not as sensitive to temperature as } \\
\text { chemical shift-based imaging }\end{array}$ \\
\hline \multirow{4}{*}{$\begin{array}{l}\text { Phase mapping of water } \\
\text { proton chemical shift } \\
\text { imaging }\end{array}$} & Very sensitive to thermal changes & $\begin{array}{l}\text { Affected by tissue motion and susceptibility } \\
\text { effects (metabolic changes, blood flow) }\end{array}$ \\
\hline & $\begin{array}{l}\text { Proton chemical shift is measured } \\
\text { independently from other parameters }\end{array}$ & $\begin{array}{l}\text { Tissue-specific temperature coefficients in } \\
\text { vivo are not well known yet }\end{array}$ \\
\hline & $\begin{array}{l}\text { Provides quantitative temperature } \\
\text { measurement }\end{array}$ & $\begin{array}{l}\text { Ineffective in measuring thermal changes in } \\
\text { low water content tissues (fat, bone } \\
\text { marrow) }\end{array}$ \\
\hline & $\begin{array}{l}\text { Short acquisition time (seconds) with fast } \\
\text { gradient-recalled echo sequences and phase } \\
\text { mapping }\end{array}$ & \\
\hline
\end{tabular}




\begin{tabular}{|c|c|c|}
\hline $\begin{array}{l}\text { 2D-Optical-Flow } \\
\text { computation of T1- } \\
\text { weighted images }\end{array}$ & $\begin{array}{l}\text { Spatio-temporal visualization and } \\
\text { prediction of thermal changes }\end{array}$ & $\begin{array}{l}\text { Computational effort (especially for 3D- } \\
\text { OF) }\end{array}$ \\
\hline & $\begin{array}{l}\text { Applicability for 3D-monitoring } \\
\text { Provides basis for automated control of } \\
\text { thermal deposition }\end{array}$ & $\begin{array}{l}\text { Not practical if } \mathrm{S} / \mathrm{N} \text {-ratio is low or if } \\
\text { tissue deformation occurs } \\
\text { Temperature dependency of } T l \text { is strongly } \\
\text { tissue specific }\end{array}$ \\
\hline
\end{tabular}

\section{Figures:}



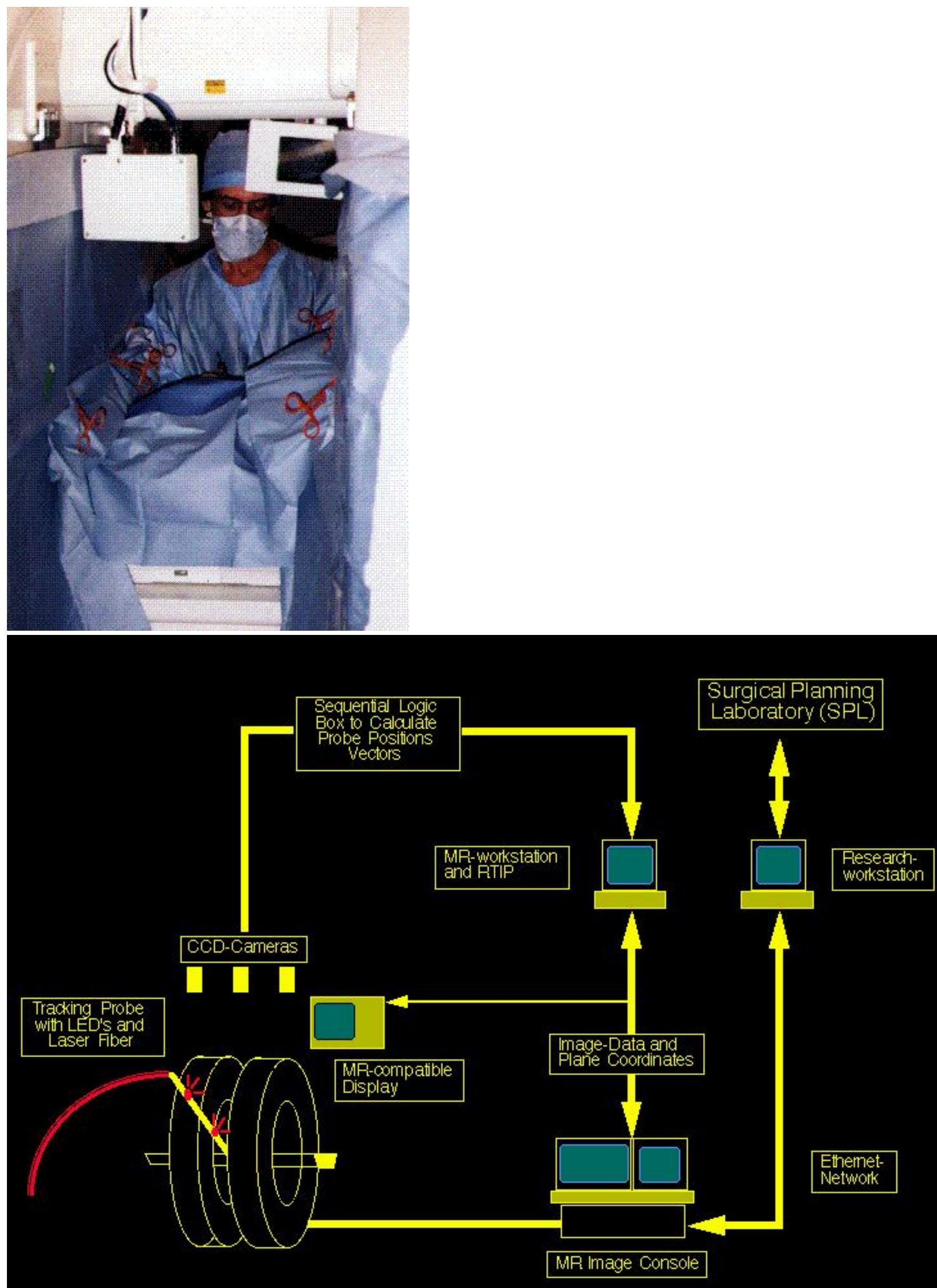
Figure 1: Performance in the Open-MR System

The open-magnet design permits direct clinical access to the patient (a) and simultaneous interactive control of the MR imaging process. Light-emitting diodes (LEDs) on a handhold probe (arrow) with the biopsy needle or laser fiber attached were registered by CCD cameras (b) providing positional information for interactive imaging and targeting tumor tissue.


65 sec
$13 \mathrm{sec}$
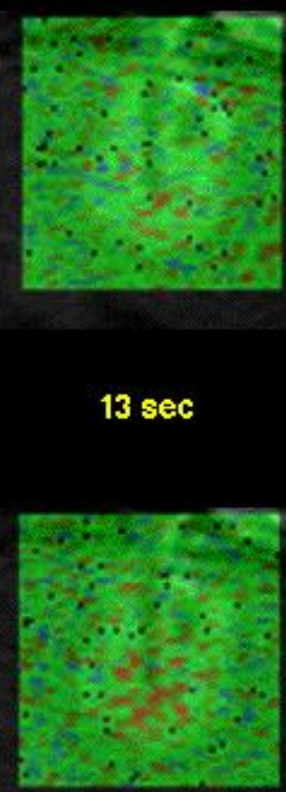

$78 \mathrm{sec}$



26 sec

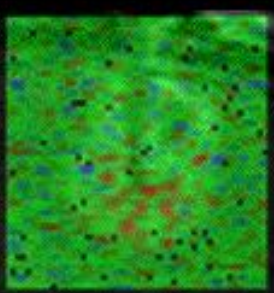

$91 \mathrm{sec}$

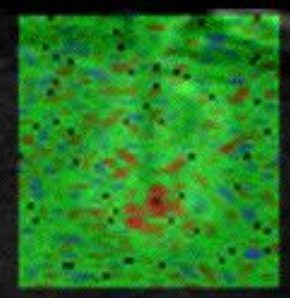

39 sec

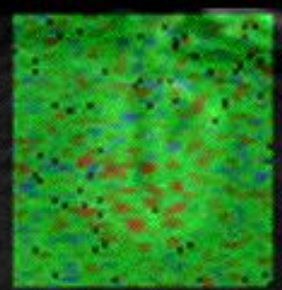

103 sec

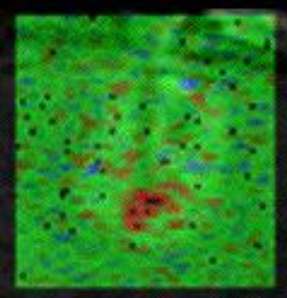

52 sec

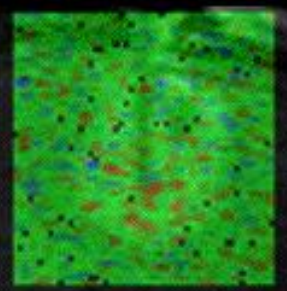

$116 \mathrm{sec}$

Figure 2: Interstitial laser treatment of a brain tumor (recurrent glioma); A user-defined ROI, drawn on the axial contrast-enhanced T1-weighted baseline image defined the region of image processing accomplished with our in-house-developed software. The resultant images, obtained every 13 seconds during and after ILT (single fiber, 4 Watt, 1 minute) were magnified and superimposed on the grayscaled image. The SI-changes are color-coded in red.




Figure 3a: Interstitial laser treatment of a brain tumor (recurrent glioma); image-processing done with the Real Time Processor. User-defined ROIs were drawn on axial T1-weighted images around the tip of the laser fiber (ROI 1) and within the white matter of the brain (ROI 2, ROI 3). During ILT (single fiber, 4 Watt, 1 minute), the amount of SI changes in the first ROI increased until the fourth calculation (approx. at the end of ILT) then decreased until imaging was completed. Within the other two ROIs, only random variations of SI changes were observed.



Figure 3b: Interstitial laser treatment of a liver tumor (metastatic leiomyosarcoma); image-processing done with the Real Time Processor. The red signal within the yellow ROI represented the color-coded SI changes between the baseline image and the image taken during therapy (single fiber, 2 Watt, 8 minutes). The graph bar on the right depicted the averaged SI changes over time within the tumor ROI (upper curve) and within a reference ROI (lower curve). The variation of SI values on the lower curve were due to slight motion artifacts, even during breathhold acquisition.

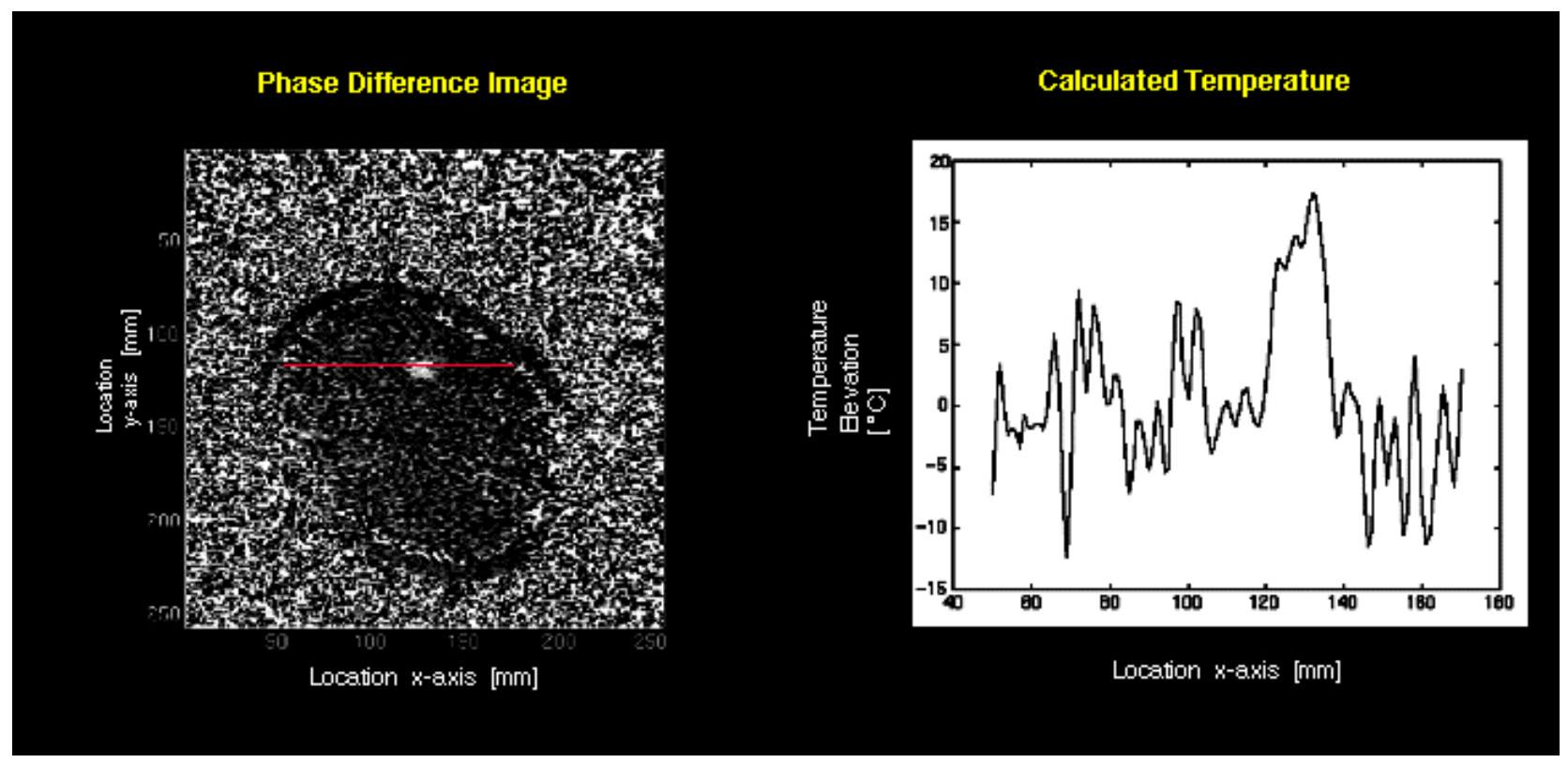

Figure 4: Chemical shift-based imaging with phase mapping of a left parietal brain tumor (recurrent glioma). On the resultant image thermal changes are highlighted within the tumor (left window) during ILT (single fiber, 3 Watt, 1 minute). Temperature gradients were calculated along the horizontal red line, 
demonstrating a temperature elevation of more than 15 degrees Celsius above body temperature (right window).

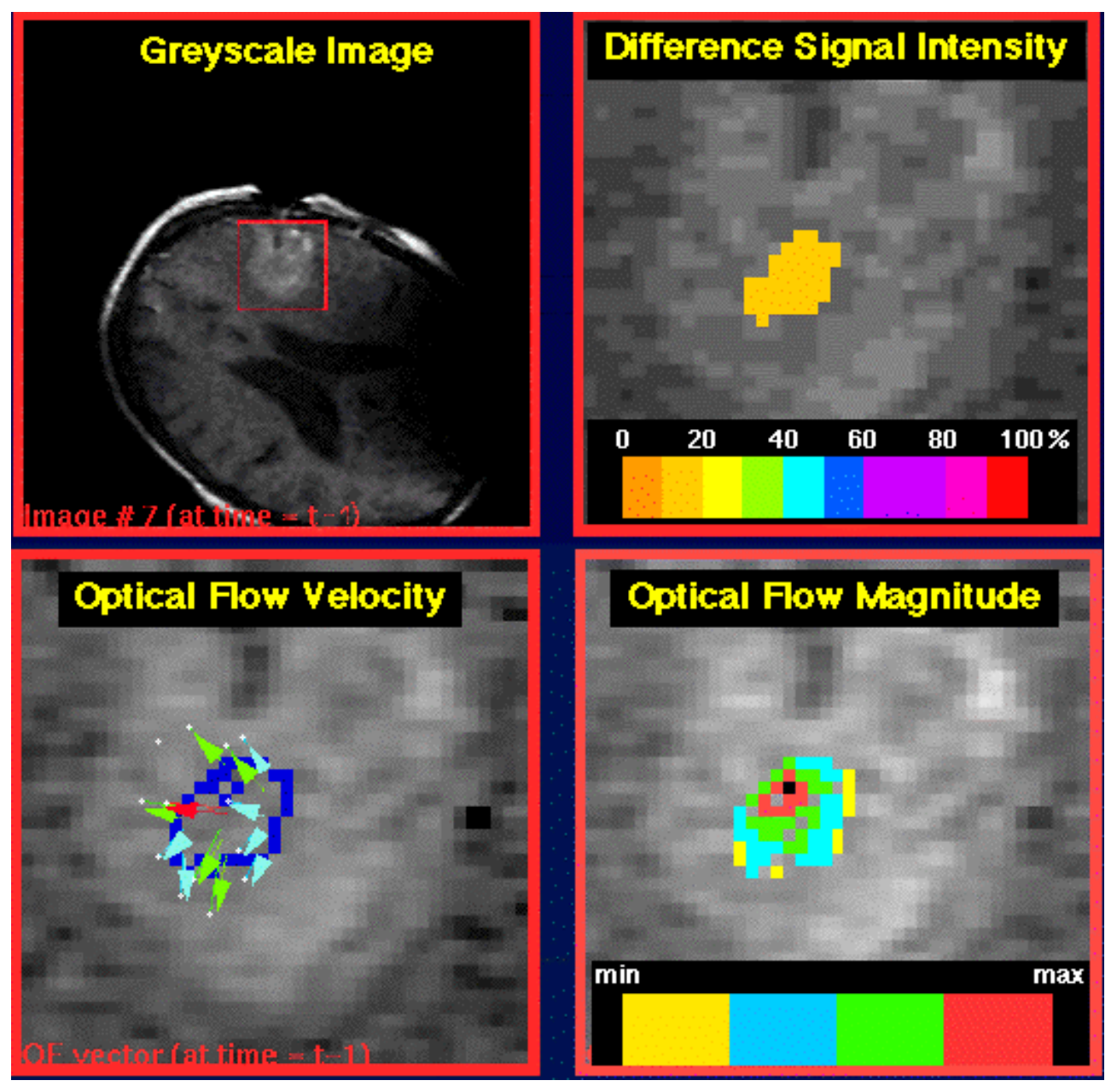

Figure 5: Monitor display of the OF computation during ILT of a brain tumor. (a) A user-chosen ROI boundary was drawn on the T1-weighted baseline image in the upper left window. The upper right window (b) displayed a magnified view of the ROI and the result of the color-coded pixel subtraction overlaid on the grayscale image at 30 seconds during laser irradiation. On the lower left window (c) the predicted OF velocity vectors were superimposed on the grayscale images, and on the lower right window (d) the predicted magnitude of OF was displayed, with color tables representing the amount of thermal change.

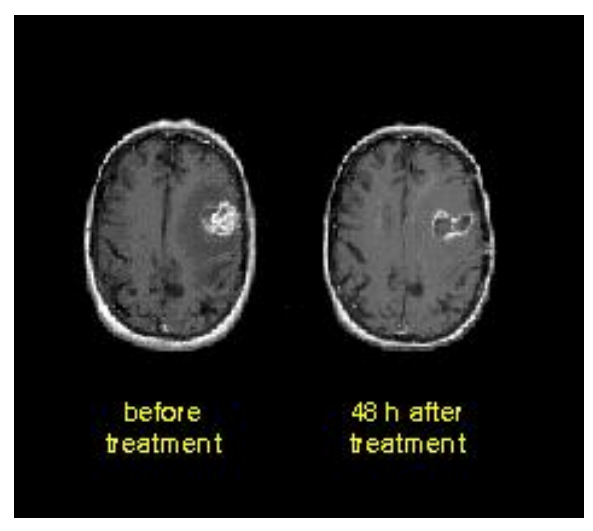

Figure 6: T1-weighted and contrast-enhanced MRI (left) before treatment demonstrated a left parietal brain tumor (diffuse astrocytoma) with a diameter of $1.2 \times 1.0 \times 0.7 \mathrm{~cm}$. With a single fiber treatment $(4 \mathrm{~W}, 6 \times 1 \mathrm{minutes}), 5 \mathrm{~mm}$ withdrawn between two lasersets, a total energy of $1440 \mathrm{~J}$ was applied and a large necrotic zone was seen 48 hours later. 

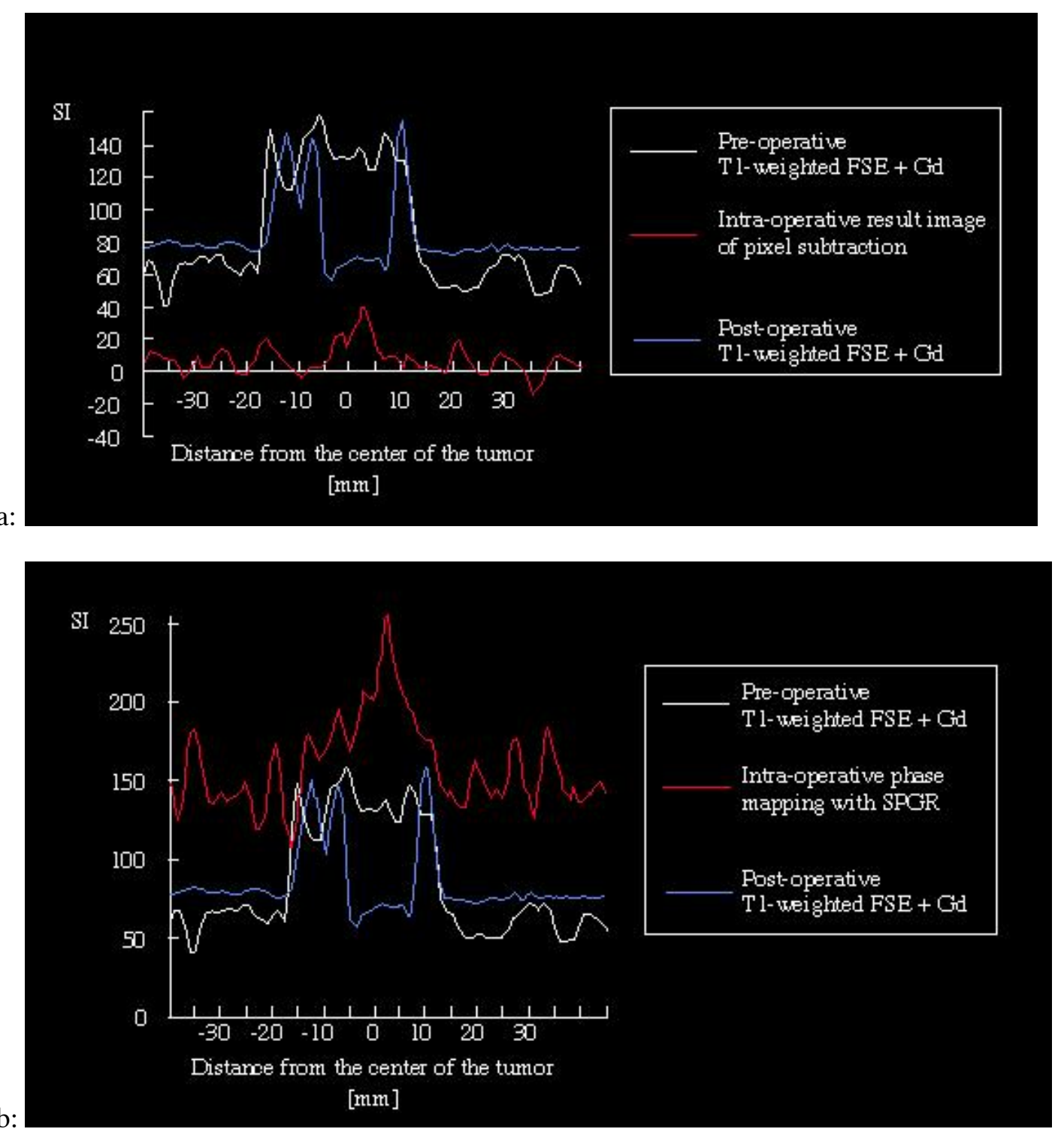

Figure 7: Line plot of SI values across a brain tumor during pixel subtraction (a) or during phase mapping (b): (a) the white line demonstrates the increased SI on the contrast-enhanced pre-operative T1-weighted image. The red line indicates the increase of the SI on the pixel-subtraction during irradiation. The blue line indicates the signal loss within a 2 $\mathrm{cm}$ large necrotic area on the follow-up image. (b) The SI of the phase difference image at the end of a laser irradiation matches with the necrotic area.

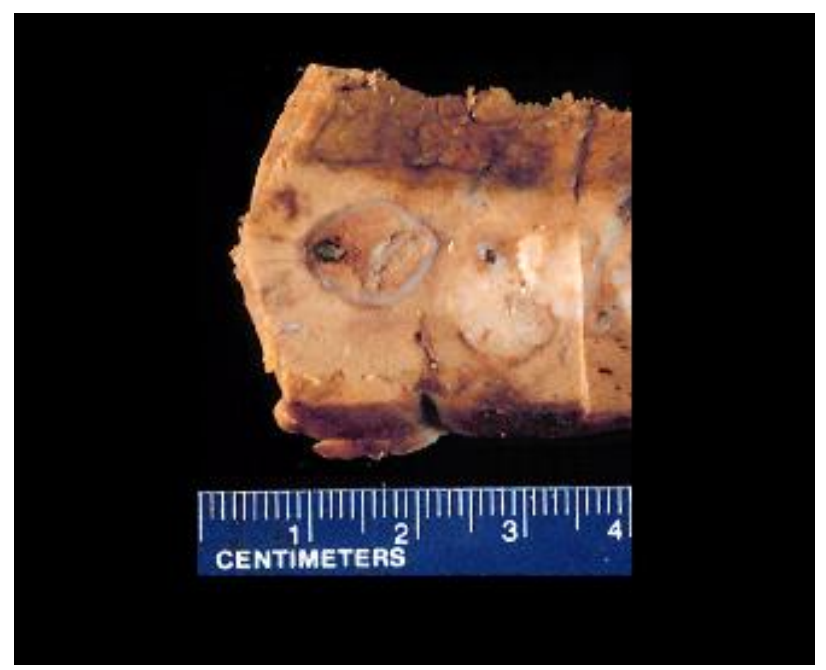


Figure 8: Specimen retrieved from partial hepatectomy. A liver metastasis (colon carcinoma) was treated with three 8minute irradiations $(2 \mathrm{~W})$; the first 8 minutes with a single fiber in place, then $2 \times 8$ minutes with 2 fibers in place. A total energy of $4880 \mathrm{~J}$ was applied. Six months later, a necrotic tumor region $(1.2 \times 1.0 \mathrm{~cm})$ was visible with a white fibrotic rim (arrow) and a small area of carbonization. 